\title{
Embracing communication
}

\author{
Although it should be a given that scholarly communication must be clear and accurate, researchers, particularly \\ those in the field of human genetics, can also promote the responsible reporting of their findings to a broader public \\ audience in ways that heighten understanding and reduce misinterpretation.
}

( ne of our main goals as a scientific journal is to promote the dissemination of research findings to the research community and beyond in an efficient and understandable manner. This is reflected in part of Nature's mission statement, which pledges "to ensure that the results of science are rapidly disseminated to the public throughout the world, in a fashion that conveys their significance for knowledge, culture and daily life". This is adapted from Nature's original mission statement that was published in 1869 when Nature was launched. This mission statement remained relevant when Nature Genetics was launched in 1992 as the first Nature Research journal, and it continues to guide us today. From the start, it has been our intention to support the communication of science, not just to the scientific community, but also to society in general.

The importance of accurately communicating science in a way that is digestible to a general audience has been acutely highlighted by the COVID-19 epidemic. When public health is at stake and there is inevitable uncertainty with regards to what we know, it is crucial that research results are not only conveyed rapidly, but also responsibly so. Across the public sphere there are different levels of scientific literacy, and during a pandemic information presented without appropriate context or caveats can be ignored, misunderstood or even weaponized by bad faith agents, to the detriment of epidemiological control measures. Of course, journalists and news sites have an enormous role to play in how science gets reported to the public. However, scientists themselves can also act as a powerful tool for communicating science to a general audience in understandable and engaging ways.

In the field of human genetics research in particular, this responsibility for trustworthy communication is especially heightened. There is a horrendous history of genetics being used to justify abhorrent attitudes, policies and violence against marginalized groups, and the whole community should be vigilant about combatting misconceptions that continue to harm people today. As genome sequencing becomes more prevalent and computational analyses become increasingly sophisticated, it can be difficult to easily translate genetic findings for a general non-science audience. This can leave reported genetic findings, such as those from genome-wide association studies (GWAS), vulnerable to incorrect interpretation or conclusions.

There are different avenues for researchers to contribute to education for the public, and one such effort is highlighted in this issue of Nature Genetics. Martschenko and colleagues introduce the FoGs database of FAQs on Genomic Studies. This open access database collects FAQ documents that accompany publication of GWAS performed on sensitive phenotypes, such as educational attainment. The GWAS FAQs serve to explain, interpret and qualify GWAS results, and the database provides a single browsable resource of these documents. The authors commend the different groups that have been writing GWAS FAQs with their studies, and they encourage researchers who are performing GWAS for sensitive phenotypes to consider including FAQs for ease of interpretation to the lay public. To assist the adoption of GWAS FAQs, they are developing a template for researchers to help them more easily generate these documents. We agree that GWAS FAQs are a useful option for the responsible reporting of genetics findings for a broad audience, allowing authors to convey in less technical language the implications of their results, including, importantly, what they do not show.

Science as an endeavor is meant to further our understanding of the natural world. In addition, the research enterprise aspires to improve our health and reduce harm to our planet, aims captured by the United Nations Sustainable Development Goals. It is our hope that researchers will continue to embrace their role as translators of their research findings so that the full promise of scientific achievement, meant to benefit all humanity, can be realized.

Published online: 7 September 2021 https://doi.org/10.1038/s41588-021-00942-8 